\title{
Cambios en índice de masa corporal en pacientes portadores de apnea del sueño a un año de tratamiento con dispositivo de presión de aire positiva continua (CPAP)
}

\author{
LUZ MARÍA TRUJILLO G.***,a, CONSTANZA SALAS C. ${ }^{a}$, ASTRID VON OETINGER G.***,****,a, \\ RODRIGO TORRES CASTRO*****,a y KABIR P. SADARANGANI***,****,a
}

Changes in body mass index in sleep apnea patients with a year of continuous positive airway pressure treatment (CPAP)

Sleep Apnea Syndrome (SAS) it is highly related to obesity. The main purpose of this study is to determine the variation between Apnea Hypopnea Index (AHI) and Body Mass Index (BMI) on sleep apnea patients after a year of CPAP treatment on the Linde Sleep Center. Results: 104 male patients were included in this study, the variables analyzed were; AHI, BMI, Epworth daytime somnolence. As for the data obtained from BMI, after one year of treatment with CPAP, the BMI showed a significant decrease $(p<0.001)$. In the daytime sleepiness scale, a significant decrease was also found between pre and post treatment values. Discussion: Evidence has consistently suggested that higher body weight would also have higher levels of AHI, and the improvements in BMI referred to in this study emphasize the importance of proper treatment not only in control of respiratory events, but in the reduction of body weight.

Key words: Sleep apnea syndrome; body mass index; continuous positive airways pressure; obesity.

\section{Resumen}

El síndrome de apnea hipoapnea del sueño (SAHS), está asociado fuertemente a la obesidad. El objetivo de este estudio es determinar las variaciones en el índice de masa corporal (IMC) en pacientes portadores de SAHS luego de un año de tratamiento con CPAP. Resultados: 104 pacientes varones fueron incluidos en este estudio, las variables analizadas fueron: índice de apnea-hipopnea (IAH), índice de masa corporal (IMC) y cuestionario de somnolencia diurna Epworth. Respecto a los datos obtenidos de IMC, se encontró tras un año de tratamiento con CPAP un descenso significativo de esta variable $(p<0,001)$. En la escala de somnolencia también se obtuvo un descenso significativo. Discusión: La evidencia ha sugerido regularmente que a mayor peso corporal existirían también niveles más elevados de IAH, las mejoras en el IMC referidas en este estudio, resaltan la importancia del correcto tratamiento no solo en el control de los eventos respiratorios, sino que en la disminución del peso corporal.

Palabras clave: Sindrome de apnea del sueño; indice de masa corporal; presión positiva continua de vías aéreas; obesidad.

\footnotetext{
* Escuela de Kinesiología, Facultad de Odontología y Salud, Universidad Diego Portales, Santiago, Chile.

** Escuela de Kinesiología, Facultad de Ciencias de la Salud, Universidad de Las Américas, Santiago, Chile.

*** Escuela de Kinesiología, Facultad Ciencias de la Salud, Universidad San Sebastián

$* * * * \quad$ Facultad de Ciencias, Universidad Mayor, Chile.

***** Departamento de Kinesiología, Facultad de Medicina, Universidad de Chile.

${ }^{\mathrm{a}}$ Kinesiólogo/a.
} 


\section{Introducción}

En las últimas décadas se ha visto un incremento de las enfermedades no transmisibles (ENT) asociadas a estilos de vida no saludables ${ }^{1}$. En el caso de Chile en adultos mayores de 15 años la obesidad ha aumentado significativamente presentándose cifras de $28,6 \%$ de obesidad en hombres y $33,7 \%$ en mujeres, lo que sitúa a nuestro país sobre los promedios mundiales ${ }^{2}$, con un $74,2 \%$ de la población adulta con sobrepeso u obesidad ${ }^{3}$.

La obesidad es una enfermedad y factor de riesgo de otras patologías, como los trastornos del sueño, y en especial el síndrome de apnea hipoapnea del sueño (SAHS) ${ }^{4-6}$. El SAHS se define como la presencia de al menos cinco episodios de apnea o hipopnea por hora durante el sueño ${ }^{7}$. La causa de este trastorno es una obstrucción de la faringe que impide la entrada de aire en los pulmones. Esta limitación en el flujo provoca desaturaciones periódicas de oxígeno, el aumento de la presión arterial y la fragmentación del sueño ${ }^{8}$. El diagnóstico y severidad del SAHS se basa en el índice de apneas-hipopneas (IAH) que es la media del número de apneas e hipoapneas por hora de sueño?.

En cuanto a la prevalencia de esta patología, Senaratna y cols. en una reciente revisión sistemática, mostró que la prevalencia mundial en población adulta mayor de 18 años es de entre 9 y $38 \%$, siendo en hombres entre 13 y $33 \%$ y en mujeres entre 6 y $19 \%$. Existen varios estudios que asocian estas variables con el efecto positivo de la edad, el género masculino y la obesidad, donde la incidencia es mayor en obesos en comparación con personas con sobrepeso ${ }^{4,6,10}$, reafirmando lo comunicado por otros autores que señalan una incidencia de SAHS menor de $70 \%$ en pacientes obesos ${ }^{5,11,12}$.

La presión positiva continua de vías aéreas (CPAP) es el tratamiento de elección para el SAHS moderado y severo, restaurando el flujo respiratorio durante el sueño, lo que resulta en una mejor saturación de oxígeno en la sangre y normalización de la arquitectura del sueño. Además, disminuye la somnolencia objetiva y subjetiva, los síntomas depresivos y mejora la atención $^{10,13}$. El objetivo de este estudio fue determinar el cambio del índice de masa corporal en sujetos con síndrome de apneas del sueño posterior el uso de CPAP.

\section{Material y Método}

Este estudio es descriptivo y retrospectivo. Se analizaron los datos obtenidos en un centro de tratamiento de trastornos del sueño de la ciudad de Santiago de Chile, de pacientes portadores de SAHS, al inicio y tras un año de tratamiento con un dispositivo de presión de aire continua (CPAP).

\section{Pacientes}

Se incluyeron los datos de los pacientes que se atienden de forma ambulatoria en el Centro de Tratamiento del Sueño Linde de Santiago de Chile. Los criterios de inclusión fueron: pacientes varones, mayores de 45 años, portadores de SAHS en sus distintos niveles, que cumplan un año de tratamiento con dispositivo de presión continua CPAP, con un mínimo de 4 horas de utilización por noche, mínimo 5 días a la semana, con diagnóstico realizado a través de polisomnografía basal acorde a los indicadores de la American Association of Sleep Medicine (AASM) ${ }^{9}$. Como criterios de exclusión se consideraron: portadores de patologías neuromusculares, patologías restrictivas de la caja torácica y/o anomalías genéticas y/o congénitas, que se hayan realizado cirugías bariátricas y/o cirugías maxilofaciales, pacientes hipertensos y/o con enfermedad pulmonar obstructiva crónica, pacientes que hayan cambiado el modo de terapia del dispositivo CPAP a BiPAP (Bilevel), que presenten interrupciones en la terapia del CPAP por más de 30 días en el año de estudio, pacientes que se hayan sometido a programas de actividades físicas, farmacológicas y/o nutricionales para bajar de peso.

\section{Datos recopilados y manejo de la información}

Los datos de los pacientes incluidos en el estudio se obtuvieron de la base de datos que maneja el Centro de Tratamiento del Sueño de la empresa Linde Gas Chile, S.A., para su análisis estadístico. Este estudio cumplió con todos los requisitos éticos y legales sobre protección de datos de carácter personal y fue aprobado por el Comité de Bioética de la Facultad de Medicina de la Universidad Diego Portales.

\section{Mediciones}

\section{a) Índice de apnea hipoapnea (IAH)}

Se evaluó la estratificación del SAHS acorde a la American Academy of Sleep Medicine (AASM) ${ }^{9}$. Considerando Normal: IAH menor o igual a 5 por hora, Leve: IAH entre 5-15 por hora, Moderado: IAH entre 15-30 por hora, Severo: IAH mayor o igual 30 por hora9. El IAH se obtuvo mediante la polisomnografía del paciente $^{14}$.

\section{b) Índice de masa corporal (IMC)}

Los datos de índice de masa corporal (IMC), fueron obtenidos de las fichas de los pacientes 
del Centro de Tratamiento del Sueño Linde S.A., entre los años 2010 al 2013, evaluados por las profesionales kinesiólogas que trabajan en el centro de tratamiento del sueño, se registraron los datos en forma controlada, midiendo peso y talla según: $\mathrm{IMC}=\mathrm{Peso} /$ Talla $^{2}\left(\mathrm{~kg} / \mathrm{m}^{2}\right)^{2}$, expresado en percentiles y como puntaje $Z(Z=$ (valormedia)/1DS). Considerando como obesidad un IMC mayor al percentil 95 para edad y sexo ${ }^{2}$. La estratificación IMC considera Normopeso: Menor a 24,99, Sobrepeso: entre 25 y 29,99, Obeso I, IMC: 30-34,99, Obeso II, IMC: 35-39,99, Obeso III o mórbido IMC mayor a $40^{2} \mathrm{~kg} / \mathrm{m}^{2}$.

\section{c) Escala de somnolencia diurna Epworth}

La Escala o cuestionario de somnolencia diurna de Epworth, es un cuestionario corto que intenta determinar o medir la somnolencia diurna $^{15,16}$. Esto puede ser de ayuda en el diagnóstico de trastornos del sueño y sirve para ver cómo influye la somnolencia en las actividades cotidianas de los pacientes ${ }^{15,16}$. La escala contempla 8 preguntas de situaciones cotidianas y probabilidad de sentir somnolencia o dormirse en alguna de ellas, con un puntaje por pregunta que va de 0 : Nunca me quedo dormido, 1: Escasa posibilidad de somnolencia, 2: moderada posibilidad de somnolencia, 3: elevada posibilidad de somnolencia. El máximo de puntos es de 24, la interpretación es la siguiente ${ }^{15,16}$ :

- Menos de 9 puntos: sin somnolencia diurna.

- Entre 10-14 puntos: leve somnolencia diurna.

- Entre 15-19 puntos: moderada somnolencia diurna.

- Entre 20-24 puntos: severa somnolencia diurna.

Los puntajes obtenidos en esta encuesta por cada paciente, antes del inicio de la terapia y al año de tratamiento, fueron obtenidos de las fichas de los pacientes del Centro de Tratamiento del Sueño Linde S.A., entre los años 2010 y 2013, el cuestionario fue aplicado por los profesionales kinesiólogos que trabajan en el centro de tratamiento del sueño, se registraron los datos en forma controlada.

\section{Análisis estadístico}

Todos los datos fueron sometidos a un procedimiento de disociación, realizándose un análisis de normalidad para las variables involucradas en el estudio. Los resultados de las variables se expresan como media aritmética \pm desviación estándar de la media para las variables con distribución normal y como mediana con rango intercuartílico para las variables no paramétricas. Según el comportamiento de normalidad de las variables, se utilizó la prueba t de Student, r de Pearson, tau-b de Kendall y prueba de asociación Chicuadrado. Se realizó un análisis de comparación entre los resultados obtenidos entre el tiempo T0 y T1, a través de la prueba no paramétrica de clasificación con signos de Wilcoxon para cada una de las variables. Para el análisis de los datos, se utilizaron los softwares estadísticos SPSS, versión 15.0 y Minitab versión 15.0.

\section{Resultados}

\section{Características de los pacientes}

El número total de pacientes considerados inicialmente fue de 957. Aplicados los criterios de inclusión y exclusión, y considerando los pacientes con los datos completos, se consideraron para este estudio a 104 pacientes (Figura 1).

Las características de los pacientes se detallan en la Tabla 1, presentándose antes del tratamiento con CPAP una edad promedio de $60,7 \pm 10,5$ años. Respecto a los datos obtenidos con el IMC el $51 \%$ de los pacientes presentaba sobrepeso, $35,6 \%$ Obesidad tipo 1 y $13,4 \%$ Obesidad tipo 2 . Respecto a las comorbilidades, el 20,2\% presentaba diabetes mellitus tipo II, el $65,4 \%$ hipertensión arterial y el 18,3\% eran fumadores.

En cuanto a la presión positiva capaz de controlar los eventos respiratorios (PEEP) esta se encontró sin distribución normal, con una mediana de 9 (8-11 $\left.\mathrm{cm} \mathrm{H}_{2} \mathrm{O}\right)$. El índice de apnea hipoapnea (IAH) presentó una mediana de 29 eventos por hora $(19,2-53,8)$. En cuanto a la escala de somnolencia diurna de Epworth, el resultado fue de $14,4 \pm 5,4$ puntos. Los pacientes con indicación de uso de CPAP fueron el $64,4 \%$ y con uso de AutoCPAP el 35,6\%.

\section{Estadística descriptiva}

Cambios pre y post tratamiento.

\section{a) Análisis variable IMC}

Respecto al IMC después de un año de tratamiento con CPAP, los resultados muestran el descenso del IMC. El descenso fue de 29,7 (28-32,7) a 27,8 (26-30), siendo este cambio estadísticamente significativo $(\mathrm{p}<0,001)$ (Tabla 2).

En cuanto a los cambios acorde al estado nutricional, los pacientes sobrepeso bajaron su IMC de $28(26,2-29)$ a $26(25-27)(\mathrm{p}<0,001)$, los obesos tipo 1 cambiaron su IMC de $32(30,8-33)$ a 30 $(27,8-30,2)(\mathrm{p}<0,001)$ quedando ambos bajo la misma clasificación. Sin embargo, los obesos tipo 2 disminuyeron de $37(35,3-37,6)$ a $30,6(29,5-$ 


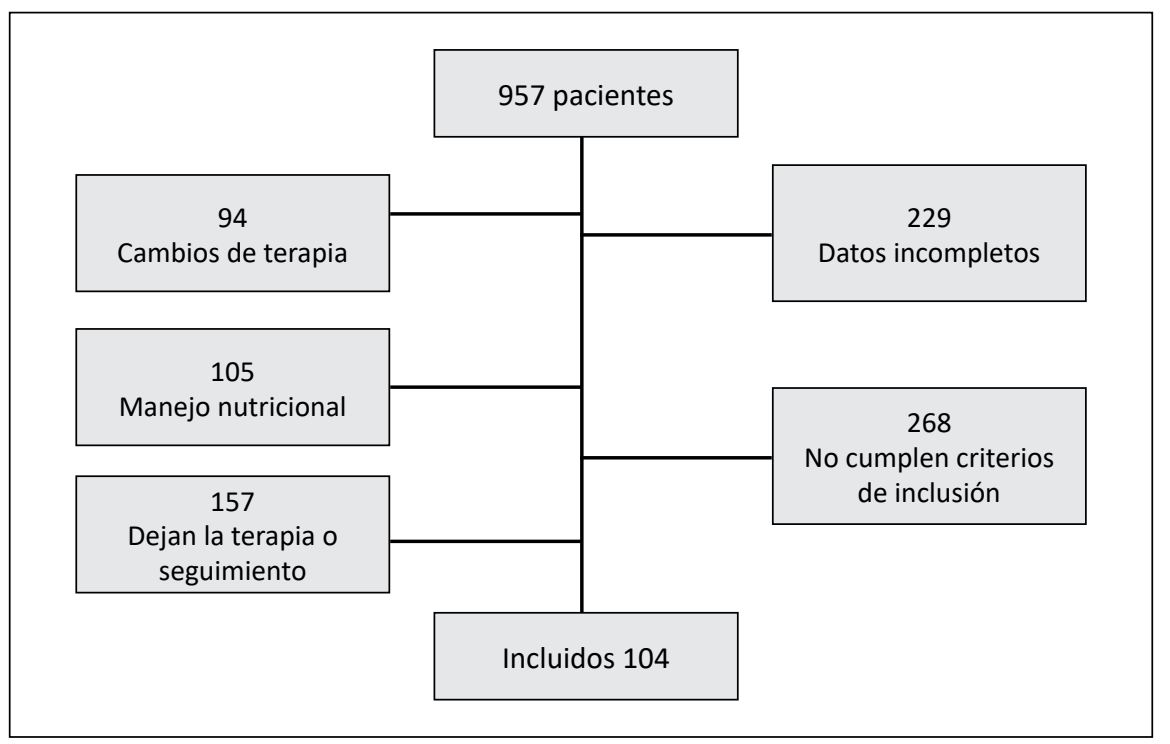

Figura 1. Diagrama de flujo para la selección de pacientes del estudio.
Tabla 1. Características de 104 pacientes con síndrome de apnea-hipopnea del sueño tratados con CPAP durante 1 año

\begin{tabular}{|lc|}
\hline Edad (años) & $60,7 \pm 10,5$ \\
PEEP $\left(\mathrm{cmH}_{2} \mathrm{O}\right)$ & $9(8-11)$ \\
IAH & $29(19,2-53,8)$ \\
Epworth & $14,5 \pm 5,4$ \\
IMC & $29,7(28-32,7)$ \\
\hline Interfase & \\
Nasal & $55(52,9 \%)$ \\
Oronasal & $49(47,1 \%)$ \\
Estado nutricional & \\
Sobrepeso & $53(51,0 \%)$ \\
Obesidad tipo 1 & $37(35,6 \%)$ \\
Obesidad tipo 2 & $14(13,4 \%)$ \\
Terapia & \\
CPAP & $67(64,4 \%)$ \\
AutoCPAP & $37(35,6 \%)$ \\
DM & \\
Sí & $21(20,2 \%)$ \\
No & $83(79,8 \%)$ \\
HTA & \\
Sí & $68(65,4 \%)$ \\
No & $36(34,6 \%)$ \\
TAB & \\
Sí & $19(18,3 \%)$ \\
No & $85(81,7 \%)$ \\
\hline
\end{tabular}

Los valores se expresan como la media \pm DE si los datos se distribuyen normalmente o como mediana (P25-P75) si la distribución de los datos es sesgada. PEEP: Presión positiva al final de la espiración por sus siglas en Inglés; Epworth: Escala de somnolencia diurna. IMC: Índice de masa corporal; CPAP: Dispositivo de ventilación mecánica no invasiva que entrega presión de aire positiva continua, por sus siglas en inglés; Auto CPAP: Dispositivo CPAP automático; DM: Diabetes mellitus; HTA: hipertensión arterial; TAB: consumo de tabaco.
Tabla 2. Índice de apnea-hipoapnea, puntaje de somnolencia de Epworth e IMC pre y post tratamiento con presión positiva continua (CPAP)

\begin{tabular}{|lccc|}
\hline & Pre & Post & p \\
IAH & $29(19,2-53,8)$ & $4,2(2,4-5,9)$ & $<0,001$ \\
Epworth & $14,5 \pm 5,4$ & $3,6 \pm 2,6$ & $<0,001$ \\
IMC & $29,7(28-32,7)$ & $27,8(26-30)$ & $<0,001$ \\
\hline
\end{tabular}

IAH: Índice de apnea hipoapnea; Epworth: Escala de somnolencia diurna; IMC: Índice de masa corporal.

35,3) su IMC, clasificándose ahora, como obeso tipo $1(\mathrm{p}=0,002)$ (Tabla 3$)$.

Respecto a los análisis de sensibilidad para comparar pacientes respondedores de no respondedores, se encontraron diferencias significativas en IMC $(p<0,001)$.

\section{b) Análisis variable IAH}

Respecto al IAH, en la Tabla 3 se observa el descenso en esta variable, pre y post tratamiento, la primera evaluación se realizó a través de la polisomnografía basal sin uso de CPAP, y la segunda evaluación con uso de CPAP.

\section{c) Puntaje Epworth}

Respecto al puntaje obtenido en la escala de somnolencia de Epworth, este varió de 14,5 \pm 5,4 a $3,6 \pm 2,6$ puntos $(\mathrm{p}<0,001)$ tras un año de tratamiento con CPAP (Tabla 2).

Los cambios acordes al estado nutricional, muestran un descenso en los puntajes obtenidos en las tres categorías de pacientes; sobrepeso, obeso tipo 1 y obeso tipo $2(\mathrm{p}<0,001)$ (Tabla 3$)$. 
Tabla 3. Efectos de 1 año de tratamiento con CPAP en las tres series de pacientes

\begin{tabular}{|lccccccccc|}
\hline & \multicolumn{3}{c}{ Sobrepeso $(\mathbf{n}=\mathbf{5 3})$} & \multicolumn{4}{c}{ Obesidad tipo 1 $(\mathbf{n}=\mathbf{3 7})$} & \multicolumn{3}{c|}{ Obesidad tipo 2 (n= 14) } \\
& Pre & Post & $\mathbf{p}$ & Pre & Post & p & & & \\
IAH & 25,2 & 3,6 & $<0,001$ & 42,7 & 4,4 & $<0,001$ & 40,6 & 4,4 & $<0,001$ \\
& $(18,2-35,5)$ & $(2,1-5,8)$ & & $(23,8-62,3)$ & $(2,5-5,8)$ & & $(19,7-59,5)$ & $(2,9-6,5)$ & \\
Epworth & $14,1 \pm 5,5$ & $3,8 \pm 2,5$ & $<0,001$ & $14,4 \pm 5$ & $3,2 \pm 1,9$ & $<0,001$ & $15,7 \pm 6,4$ & $4,3 \pm 4,1$ & $<0,001$ \\
IMC & 28 & 26 & $<0,001$ & 32 & 30 & $<0,001$ & 37 & 30,6 & 0,002 \\
& $(26,2-29)$ & $(25-27)$ & & $(30,8-33)$ & $(27,8-30,2)$ & & $(35,3-37,6)$ & $(29,5-35,3)$ & \\
\hline
\end{tabular}

IAH: Índice de apnea hipoapnea; Epworth: Escala de somnolencia diurna; IMC: Índice de masa corporal.

\section{Discusión}

Los principales hallazgos de este estudio indican que pacientes varones, portadores de apnea del sueño analizados en este estudio, posterior a un año de tratamiento con CPAP presentan una disminución significativa de su IMC. Lo anterior es apoyado por la literatura actual sobre la relación que existe entre el SAHS y la obesidad ${ }^{12,17-20}$.

Existe bastante evidencia científica actualizada que asocia el mayor peso corporal con niveles más elevados de IAH en los pacientes portadores de SAHS $^{18}$. Distintos estudios han mostrado que los pacientes con SAHS son más propensos a subir de peso en comparación a sujetos obesos que no presentan trastornos del sueño ${ }^{21}$, un estudio realizado con 773 pacientes con SAHS dio como resultado, que el $75 \%$ de pacientes con SAHS, presentan obesidad (IMC $>30)^{17}$. Mahat y col demostraron que, en los pacientes expuestos a hipoxia aguda por apnea del sueño, la actividad de la lipoproteína lipasa se encuentra disminuida hasta en 6 veces, lo que también afecta el correcto control del peso en este tipo de pacientes ${ }^{22}$.

La asociación entre SAHS y obesidad responde a muchos factores, la severidad del SAHS se ha relacionado con el $\mathrm{IMC}^{7,23}$, dentro de los factores involucrados en esta asociación están el aumento de IL-6 y del factor de necrosis tumoral (TNF) en pacientes obesos ${ }^{25}$, lo que aumenta la somnolencia diurna ${ }^{9}$; también se ha demostrado que los pacientes con apnea del sueño asociado a obesidad, presentan niveles de Leptina y Grelina aumentados, lo que altera la saciedad y apetito $^{25,26}$, estos niveles hormonales disminuyen después de 8 meses de tratamiento con CPAP aunque el IMC no se haya modificado ${ }^{14}$. Otro estudio demostró que la cantidad de grasa visceral (predictor de riesgo cardiovascular) ${ }^{18}$ disminuye significativamente en pacientes con SAHS a los 6 meses de tratamiento con CPAP, sin pérdida de peso corporal ${ }^{27}$.

Un reciente estudio publicado por Shechter y cols en abril de 2017, concluye después de analizar a 264 adultos, que la baja de IMC en pacientes con SAHS genera mayor impacto metabólico que en los descensos en el IHA ${ }^{28}$.

Los resultados obtenidos en nuestro estudio difieren a lo observado por Myllylä y cols en el año 2016, donde la evaluación se realizó en 1.023 pacientes, con un periodo de seguimiento de 5 años, ellos concluyeron que el peso no cambió significativamente en los pacientes tratados con CPAP, pero sí se pudo observar en un $90 \%$ de los pacientes, que la ganancia de peso fue más lenta que la población general de la misma edad, y un $10 \%$ de los pacientes, a pesar de la alta adherencia al CPAP, continuaron con el aumento de peso $^{29}$.

Debemos considerar el alto nivel de obesidad en la población chilena y el aumento exponencial que ha tenido esta patología y su relación con otras enfermedades ${ }^{30,31}$. Además de la baja cantidad de estudios realizados en nuestra población, con nuestro estudio se refuerza y valida el impacto del correcto diagnóstico, tratamiento y seguimiento de esta patología en la población chilena.

Se incorporó en nuestro estudio solo a pacientes de género masculino, lo que puede considerarse una limitación, este motivo de exclusión fue principalmente por la edad de las pacientes mujeres que se atendían en el centro en el periodo estudiado, ya que la mayoría estaba entrando en cambios hormonales típicos de la menopausia, lo que genera cambios en el IMC, distribución de la grasa corporal y otros cambios fisiológicos que podrían influir en los resultados de este estudio ${ }^{32}$.

Como sugerencia para futuros estudios, se recomienda analizar relaciones incorporando medidas adicionales al CPAP, como lo son la actividad física que tiene alta evidencia de ser beneficiosa y favorecer aún más el descenso del IMC en estos pacientes $^{33-36}$.

Cabe resaltar que no se reportaron conflictos de interés entre los participantes de este estudio. 


\section{Agradecimientos}

Al equipo de Profesionales Centro de Tratamiento del Sueño de la empresa Linde Gas Chile S.A.

\section{Bibliografía}

1.- NATIONAL INSTITUTE OF CHILEAN STATISTICS (IND) 2012. National Survey of Physical Activity Habits. (Consultado el 9 de mayo de 2017).

2.- WHO. World Health Statistics 2014. (Consultation date 09-05-2017).

3.- MINSAL. Ministry of Health. Chile's government. National Health Survey 2016-2017 ENS Chile. Chile 2017. (Consultado el 12 de mayo de 2018).

4.- RODRÍGUEZ HERMOSA JL, ÁLVAREZ-SALA WATHER JL, CALLE RUBIO M, FERNÁNDEZ SÁNCHEZ JM, MARTÍNEZ CRUZ R. Obstructive sleep apnea national health system. Complutense University. Madrid. Inf Ter Sist Nac Salud 1999; 23: 121-31.

5.- VGONTZAS AN, PAPANICOLAOU DA, BIXLER EO, HOPPER K, LOTSIKAS A, LIN HM, et al Sleep Apnea and Daytime Sleepiness and Fatigue: Relation to visceral Obesity, Insulin Resistance, and Hypercytokinemia. J Clin Endocrinol Metab 2000; 85: 1151-8.

6.- SMITH R, RONALD J, DELAIVE K, WALLD R, MANFREDA J, KRYGER MH. What are obstructive sleep apnea patient being treated for prior to this diagnosis? Chest 2002; 121: 164-72.

7.- TUOMILEHTO H, SEPPA J, UUSITUPA M. Obesity and obstructive sleep apnea-Clinical significance of weight loss. Sleep Medicine Reviews 2013; 17:321-9.

8.- Giles TL, Lasserson TJ, Smith B, White J, Wright JJ, Cates CJ. Continuous positive airways pressure for obstructive sleep apnea in adults. Cochrane Database Syst Rev 2006; 3: Art. CD001106.

9.- KAPUR VK, AUCKLEY DH, CHOWDHURI S, KUHLMANN DC, MEHRA R, RAMAR K, et al Clinical Practice Guideline for Diagnostic Testing for Adult Obstructive Sleep Apnea: An American Academy of Sleep Medicine Clinical Practice Guideline. J Clin Sleep Med 2017; 13: 479-504.

10.- SENARATNA CV, PERRET JL, LODGE CJ, LOWE AJ, CAMPBELL BE, MATHESON MC, et al Prevalence of obstructive sleep apnea in the general population: A systematic review. Sleep Medicine Reviews 2016; 2016: 1-12.

11.- PUNJABI NM. The epidemiology of adult obstructive sleep apnea. Proceedings of the American Thoracic Society 2008; 5:136-43.

12.- KIM JH, KOO YC, CHO HJ, KANG JW. Relationship between various anthropometric measures and apneahypopnea index in Korean men. Auris Nasus Larynx 2017; 2017: 1-6.
13.- HARSCH IA, KONTUREK PC, KOEBNICK C, KUEHNLEIN PP, FUCHS FS, SCHAHIN S, et al. Leptin and Ghrelin levels in patients with obstructive sleep apnoea: effect of CPAP treatment. Eur Resp J 2003; 22: 251-7.

14.- KUSHIDA C, LITTNER M, MORGENTHALER T, ALESSI C, BAILEY D, COLEMAN J, et al. Practice Parameters for the Indications for Polysomnography and Related Procedures. Sleep: 2005; 28:499-521.

15.- LONGWORTH H, MCCALLIN K, NARAYANAN RP, TURNER MA, QUENBY S, RYCROFT D, et al Screening methods for obstructive sleep apnoea in severely obese pregnant women. Clin Obes 2017; 7: 239-44.

16.- JANSSEN KC, PHILLIPSON S, O'CONNOR J, JOHNS MW. Validation of the Epworth Sleepiness Scale for Children and Adolescents using Rasch analysis. Sleep Med 2017; 33:30-5.

17.- PHILLIPS BG, HISEL TM, KATO M, PESEK CA, DYKSEN ME, NARKIEWICS K, et al Recent weight gain in patients with newly diagnosed obstructive sleep apnea. J Hypertens 1999; 17: 1297-1300.

18.- BJORNTORP P. Body fat distribution, insulin resistance, and metabolic diseases. Nutrition 1997; 13:795-803.

19.- BARCELÓ A, MORELL-GARCÍA D, SALORD N, ESQUINAS C, PÉREZ G, PÉREZ A, et al. A randomized controlled trial: branched-chain amino acid levels and glucose metabolism in patients with obesity and sleep apnea. J Sleep Res.2017; 17:1-9.

20.- KARKINSKI D, GEORGIEVSKI O, DZEKOVA-VIDIMLISKI P, MILENKOVIC T, DOKIC D. Obstructive Sleep Apnea and Lipid Abnormalities. Clinical Science 2017; 5: 19-32.

21.- BONNET M, CARLEY D, CARSKADON M, EASTON P, GUILLEMINAULT C, HARPER R, et al EEG arousals: scoring rules and examples. Sleep 1992; 15: 174-82.

22.- MAHAT B, CHASSÉ E, MAUGER JF, IMBEAULT P. Effects of acute hypoxia on human adipose tissue lipoprotein lipase activity and lipolysis. J Transl Med 2016; 14: 212-21.

23.- VICENTE-HERRERO MT, CAPDEVILA GARCÍA L, BELLIDO CAMBRÓN MDC, RAMÍREZ IÑIGUEZ DE LA TORRE MV, LLADOSA MARCO S. Cardiovascular risk and obesity in sleep apnea syndrome assessed with the Stop-Bang questionnaire. Endocrinol Diabetes Nutr 2017; 64: 544-51.

24.- VGONTZAS AN, PAPANICOLAOU DA, BIXLER EO, KALES A,TYSON K, CHROUSOS GP. Elavation of Plasma Cytokines in disorders of excessive daytime Sleepiness: Rol of Sleep Disturbance and Obesity. J Clin Endocrinol Metab 1997; 82: 1331-6.

25.- SPIEGEL K, TASALI E, PENEV P, VAN CAUTER E. Sleep curtailment in healthy young men is associated with decreased leptin levels, elevated ghrelin levels, and increased hunger and appetite. Ann Intern Med 2004; 141:846-50. 
26.- ZHANG P, LIU J, LONG S, XIE, GUO Y. Association between continuous positive airway pressure and changes in serum leptin in patients with obstructive sleep apnea: a meta-analysis. Sleep and Breathing. 2014; 18: 695-702.

27.- CHIN K, SHIMZU K, NAKAMURA T, NARAI N, HIROAKI MASUZAKI H, OGAWA Y, et al. Changes in intra-abdominal visceral fat and serum leptin levels in patients with obstructive sleep apnea syndrome following nasal continuous positive airway pressure therapy. Circulation 1999; 100: 706-12.

28.- SHECHTER A, FOSTER GD, LANG W, REBOUSSIN DM, ST-ONGE MP, ZAMMIT G, et al. Effects of a lifestyle intervention on REM sleep-related OSA severity in obese individuals with type 2 diabetes. J Sleep Res 2017; doi: 10.1111/jsr.12559.

29.- MYLLYLÄ M, KURKI S, ANTTALAINEN U, SAARESRANTA T, LAITINEN T. High Adherence to CPAP Treatment Does Not Prevent the Continuation of Weight Gain among severely Obese OSAS Patients. J Clin Sleep Med 2016; 12: 519-28.

30.- MORGENSTERN M, WANG J, BEATTY N, BATEMARCO T, SICA AL, GREENBERG H. Obstructive
Sleep Apnea: An Unexpected Cause of Insulin Resistance and Diabetes. Endocrinol Metab Clin North Am. 2014; 43: 187-204.

31.- KNUTSON KL, SPIEGEL K, PENEY P, VAN CAUTER E. The metabolic consequences of sleep deprivation. Sleep Medicine Reviews 2007; 11: 163-78.

32.- PROIETTO J. Obesity and weight management at menopause. Aust Fam Physician 2017; 46: 368-70.

33.- IFTIKHAR I, KLINE C, YOUNGSTEDT S. Effects of exercise training on sleep apnea; a meta-analysis. Lung 2014; 192: 175-84.

34.- KELLEY GA, KELLEY KS. Exercise and sleep: a systematic review of previous meta-analyses. J Evid Based Med 2017; 10: 26-36.

35.- ARAGHI MH, CHEN YF, JAGIELSKI A, CHOUDHURY S, BANERJEE D, HUSSAIN S, et al. Effectiveness of lifestyle interventions on obstructive sleep apnea (OSA): systematic review and meta-analysis. Sleep 2013; 36: 1553-62 E.

36.- KUBITZ KA, LANDERS DM, PETRUZZELLO SJ, HAN M. The effects of acute and chronic exercise on sleep. A meta-analytic review. Sports Med 1996; 21: 277-91.
Correspondencia a:

Luz María Trujillo Gittermann

Escuela de Kinesiología, Facultad de Odontología y

Salud, Universidad Diego Portales, Santiago, Chile.

Email: luzmariatrujillo.aga@gmail.com 